\title{
Controlled electromigration and oxidation of free-standing copper wires
}

\author{
J. S. Hauser ${ }^{1} \cdot$ J. Schwichtenberg $^{1}$ - M. Marz ${ }^{1} \cdot$ C. Sürgers $^{1} \cdot$ A. Seiler ${ }^{1}$ \\ U. Gerhards ${ }^{2} \cdot$ F. Messerschmidt ${ }^{2} \cdot$ A. Hensel ${ }^{2} \cdot$ R. Dittmeyer ${ }^{2} \cdot$ H. v. Löhneysen ${ }^{1,3}$. \\ R. Hoffmann-Vogel ${ }^{1,4}$
}

\begin{abstract}
We have studied controlled electromigration (EM) in free-standing copper wires. Besides electrical characterization by voltage-current measurements, structural analyses have been performed by means of scanning electron microscopy and cross-sectional microprobe measurements. We have found that oxidation during the EM in air stabilizes the free-standing wire against uncontrolled blowing, making it possible to thin the conductive part of the wire down to a conductance of a few conductance quanta $G_{0}=2 e^{2} / h$. The decisive influence of oxidation by air on the EM process was confirmed by control experiments performed under ultra-high vacuum conditions. In line with these findings, free-standing Au wires were difficult to thin down reproducibly to a conductance of a few $G_{0}$. Estimates of the local temperature in the free-standing wire are obtained from finite element method calculations.
\end{abstract}

\section{Introduction}

Electromigration (EM), i.e., the directed diffusion of metal ions in the presence of an electric current, is well known as one of the main failure mechanisms of

M. Marz

Michael.Marz@kit.edu

1 Physikalisches Institut, Karlsruhe Institute of Technology, 76049 Karlsruhe, Germany

2 Institut für Mikroverfahrenstechnik, Karlsruhe Institute of Technology, 76344 Karlsruhe, Germany

3 Institut für Festkörperphysik, Karlsruhe Institute of Technology, 76021 Karlsruhe, Germany

4 Present Address: Institut für Angewandte Physik, Karlsruhe Institute of Technology, 76049 Karlsruhe, Germany microelectronic devices [1, 2]. The EM process in a metallic wire can be controlled by applying cyclic voltage ramps during which Joule heating and/or EM is monitored by a resistance change $[3,4]$. The cycle is stopped when a preset resistance change is reached, and a new cycle is started at a lower bias. The increase of the resistance due to Joule heating is usually reversible. In addition to this reversible change, Joule heating enhances diffusion of material as the temperature increases. The EM forces (Coulomb and wind force) acting on the metal ions lead to a directional diffusion which gives rise to a thinning of the metal wire, and therefore to an irreversible increase of the resistance. This method of controlled EM is widely used to produce nanometer-sized gaps in metallic leads that can be used as electrodes to contact individual molecules [5, 6]. Up to now, mostly Au wires and contacts have been investigated. Here, we have studied the effect of controlled EM on the electric and structural properties of macroscopic, free-standing $\mathrm{Cu}$ wires. Despite the initially macroscopic diameter of the wires of $\sim 20 \mu \mathrm{m}$, we were able to thin the wires in a controlled fashion under ambient conditions down to thicknesses of a few atom diameters before breakage as inferred from the observation of conductance plateaus of a few conductance quanta. In addition, we performed electron microprobe analysis of the wire cross section close to the location where the wire is thinned by EM. The experiments demonstrate that an oxide layer forms at the perimeter of the wire during EM in air which stabilizes the free-standing wire and allows for continued thinning. The decisive effect of an oxide film surrounding the wires on the contact stability was also demonstrated by control experiments performed in ultra-high vacuum (UHV) in which free-standing wires do not show this self-stabilization effect. 


\section{Experimental}

Controlled EM was applied to free-standing $\mathrm{Cu}$ wires of several mm length with diameters between $d=15$ and $25 \mu \mathrm{m}$. Some of the wires were coated with an insulating polymeric layer. The insulating layer burnt away during the EM process and did not show any significant influence on the EM process as inferred from the overall similarity of the current-voltage characteristics. The $\mathrm{Cu}$ wires were suspended while subjected to EM. They were either soldered to two pins of a circuit board or mounted on a 'direct current sample plate' used in UHV systems (Omicron NanoTechnology GmbH, Germany). In the latter case, silver paste was used to stabilize the electric contact. Controlled EM was performed mainly under ambient condition. For comparison, some experiments were done in UHV. All EM measurements were taken in a two-terminal setup with a source meter (Keithley Model 2400) that was computer-controlled by a home-made software (LabView). For standard scanning electron microscopy (SEM) imaging, the samples were kept in the holder where EM had been performed. The images have been taken with a Zeiss Supra 55 VP SEM at an acceleration voltage between 3 and $5 \mathrm{kV}$. For cross-sectional SEM analysis, some wires were embedded in Araldite epoxy (Huntsman Advanced Materials) and then polished. The polished cross sections of the pristine wires and wires after EM were examined with a field emission microprobe Jeol JXA-8530 F with windowless wavelength-dispersive spectrometers (WDS). The images with back-scattered electrons and the elemental maps for the elements copper and oxygen were taken at a voltage of 15 and $20 \mathrm{kV}$, yielding a quantitative chemical analysis of the wire cross sections with a lateral resolution of $\leq 1 \mathrm{~m}$.

\section{Results and discussion}

\subsection{Electromigration}

Figure 1 shows the full sequence of current-voltage cycles obtained during an individual EM protocol of a free-standing $\mathrm{Cu}$ wire $(d=20 \mu \mathrm{m})$. The whole process can be separated into three stages. During the first stage, marked A, the cycles show reversible resistance changes upon increasing the bias. The non-ohmic (nonlinear) behavior in these cycles is caused by Joule heating, which in metals leads to an increase of the resistance. During the second stage $\mathrm{B}$, an irreversible increase of the resistance is observed indicating the onset of EM [3]. In this case, the end points of the individual cycles result in a belly-shaped envelope. This shape of the envelope is tentatively ascribed to a partial oxidation of the free-standing wire where

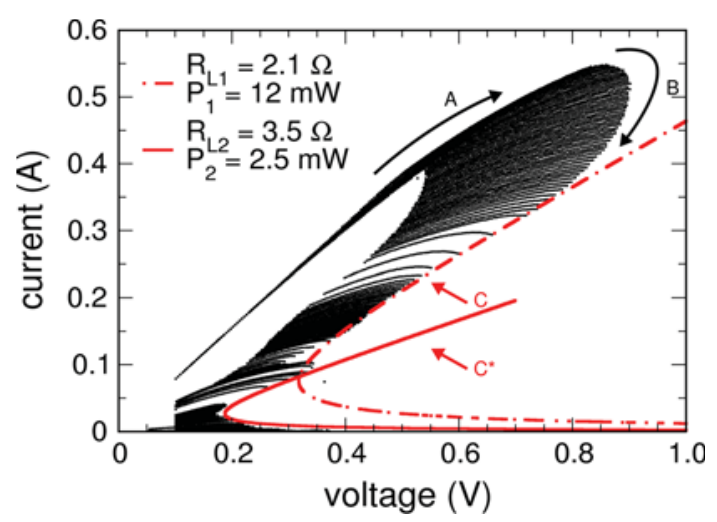

Fig. 1 Current versus voltage characteristics of the full EM process for a free-standing $20-\mu \mathrm{m} \mathrm{Cu}$ wire. The whole process can be separated into three stage. See text for details

oxidation reduces the conductive cross section of the wire. By continuing the controlled EM process, in a third region denoted as $\mathrm{C}$, the expected characteristic behavior for controlled EM is observed [7]. In this region, the end points of the cycles can be described by a hyperbolic envelope, corresponding to a curve of constant-power dissipation in the contact region, under the assumption of a constant lead resistance [4, 7]. Typically, we find two or more hyperbolic envelopes in our experiments, which we ascribe to the formation of 'hot spots' in the wire [8]. In Fig. 1, a second envelope denoted by $\mathrm{C}^{*}$ is indeed observed. The constant-power fits can be calculated from $V(I)=R_{\mathrm{L} x} \cdot I+P_{x} / I$, where $R_{\mathrm{L}}$ is the lead resistance and $P$ the dissipated power. The two constant-power lines in Fig. 1 are given by $P_{1}=12 \mathrm{~mW}, R_{\mathrm{L} 1}=2.1 \Omega$ and $P_{2}=2.5 \mathrm{~mW}, R_{\mathrm{L} 2}=3.5 \Omega$. The obtained values for the lead resistance $R_{\mathrm{L} x}(x=1,2)$ are reasonable for our samples and setup. The increase of $R_{\mathrm{L}}$ from the first to the second envelope is expected, since parts of the preformed contact will in the following contribute to the lead resistance. While the diameter of the contact decreases with continued EM, the power $P$ necessary to perform a change in the remaining contact also becomes smaller, a behavior that is reflected in our fit parameter. The overall features of the EM process with the three stages described above are highly reproducible. Approximately, 90\% of our experiments with thin free-standing $\mathrm{Cu}$ wires under ambient conditions show this behavior.

For some samples, we observe that, when the EM process is continued further, the conductive part of the wire is reduced until a few conduction channels only are left. From the measured current-voltage data $I_{i}\left(V_{i}\right)$, we determine the conductance as $G_{i}=I_{i} / V_{i}$ for each measured data point. Figure 2 shows the histogram of conductances derived from the EM data of Fig. 1 in the range $G \leq 10 G_{0}$, i.e., very close to the abscissa. Here, $G_{0}=2 e^{2} / h$ is the conductance quantum. The broad peaks indicate that particular 


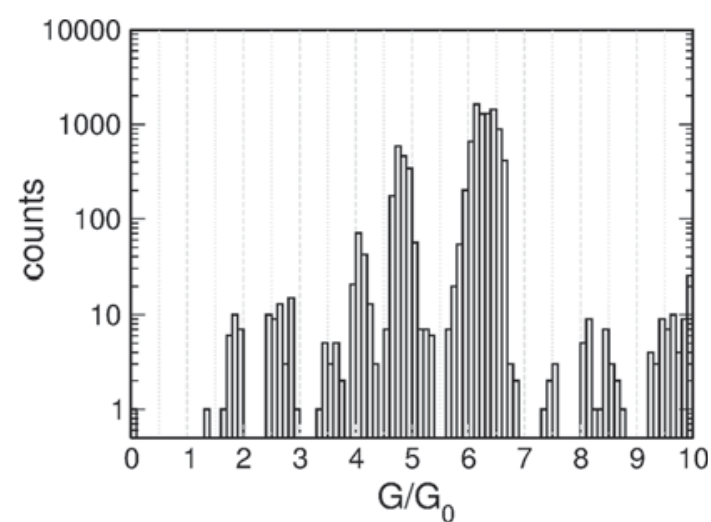

Fig. 2 Conductance histograms of the data of Fig. 1 in the lowconductance region $\left(G \leq 10 \cdot G_{0}\right)$. Note the logarithmic vertical scale

conductance values and their corresponding contact configurations are more stable, i.e., they are observed more frequently than others. We emphasize that the measurement algorithm used in Fig. 1 results in a large amplification of those stable peaks and that the peaks are generated at single bias values, i.e., not during cycles. Usually, the spacing of the peaks is on the order of $G_{0}$. The occurrence of discrete peaks and their regular spacing is typical for EM or mechanically controlled break-junction (MCBJ) contacts close to breakage where the details depend on the metals investigated [9]. For $\mathrm{Cu}$ contacts, usually a peak at just below $1 G_{0}$ and an additional one below $2 G_{0}$ are observed under different experimental conditions [10-12]. The presence of additional peaks varies for different experiments. For $\mathrm{Au}$, additional peaks above $2 G_{0}$ were found in MCBJ experiments [13, 14]. Their presence and conductance values depend on the detailed atomic structure in the junctions [9]. These additional peaks could be seen in MCBJ experiments for certain orientations of the wires only. Apparently, a structure of the contact close to equilibrium facilitates their presence. We recall that with MCBJ technique generally contacts are formed by atomic-scale 'cold-working' far from thermal equilibrium. In line with this argument, the routine observation of additional peaks in electromigrated wires that are heated, as opposed to break junctions, lends credit to this conjecture [4]. This idea should be further checked by additional experiments with better statistics.

Besides the controlled thinning process, the time dependence of the conductance at constant bias is of interest. The mean time to failure due to EM is phenomenologically given by $t_{\mathrm{F}}=A j^{-n} \exp \left(Q / k_{\mathrm{B}} T\right)$, where $A$ is a constant related to the geometry of the wires, $j$ the current density, $n$ a model parameter, $Q$ the (bias-dependent) activation energy, $k_{\mathrm{B}}$ the Boltzmann constant, and $T$ the temperature [1]. This phenomenological equation is valid for simple geometries where $n$ is
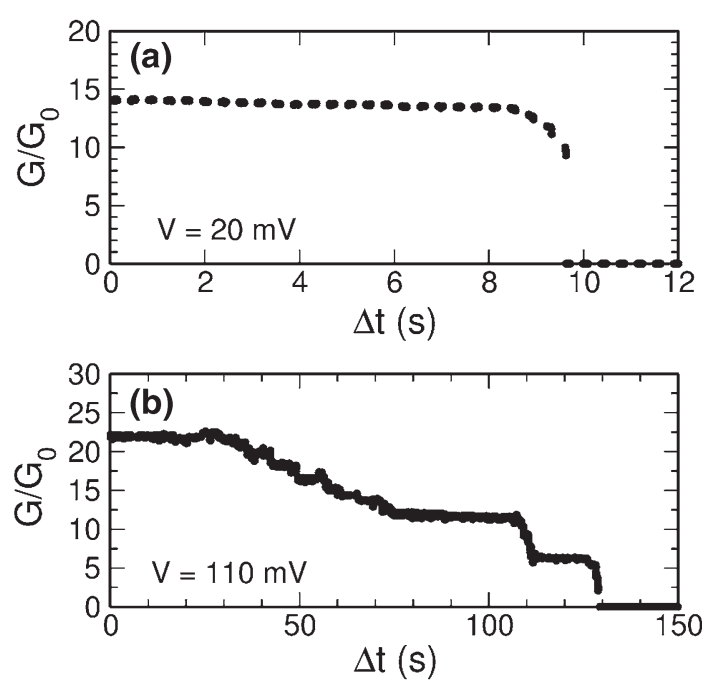

Fig. 3 Final burn-through of two different electromigrated freestanding $\mathrm{Cu}$ wires. a Sudden breakdown $(V=20 \mathrm{mV})$. b The wire breaks down in discrete steps $(V=110 \mathrm{mV})$

determined by the details of void formation and modification [15]. Subsequently, there have been many theoretical investigations on EM, especially for integrated circuits [16, 17]. However, the main dependencies, namely on temperature and current density, remain unaffected in these models. For macroscopic wires at low current densities and temperatures, $t_{\mathrm{F}}$ is usually of the order of years $[18,19]$. However, when the wire is thinned down to a cross section containing a few atoms only resulting in a conductance range of a few $G_{0}$, the constriction is mechanically destabilized, and the failure takes place on a much shorter timescale. To investigate the time dependence of the conductance, we stopped the controlled EM process when the conductance $G$ was in the range between 10 and $30 \cdot G_{0}$. Subsequently, the conductance $G(t)$ was monitored at constant applied bias. Figure 3 gives two examples of the observed time dependences. For most samples, the conductance drops abruptly so that the current falls below the noise level ( $\approx 100 \mathrm{nA})$ of the setup adapted for high applied currents ( $\sim 1 \mathrm{~A})$, as shown in Fig. 3a. This process happens faster than the acquisition time for individual data points (a few ms) even at low bias, i.e., small current. In this example, the sample bias was set to $V=20 \mathrm{mV}$. The sudden rip-off could be attributed to the forces acting on the free-standing wire, due to mechanical stress. In contrast, a few samples show a stepwise decrease of the conductance as shown in Fig. 3b. In this case, apparently a stepwise rearrangement of a few atoms only occurs in the contact region. It is worth noting that no systematic dependence of the fast or slow rupture on the applied bias was found. 


\subsection{Scanning electron microscopy}

So far, we have discussed the effect of EM on the freestanding wires with respect to their electronic properties. In the following, we focus on the structural aspects. Although the conductance value is close to or, in fact, zero after the EM process, the free-standing wires are still mechanically connected, but have become brittle and can break easily. To analyze the structural changes of the samples, a few wires were studied by scanning electron microscopy (SEM).

Figure 4 shows SEM images of different free-standing $\mathrm{Cu}$ wires at different stages of the EM process. Figure $4 \mathrm{a}$ shows the image of a free-standing $\mathrm{Cu}$ wire that was not subjected to EM. The wire shows an overall smooth surface with only few trenches along the wire (one example is marked by an arrow), most likely due to the pulling during the production process. After EM, the surface structure is
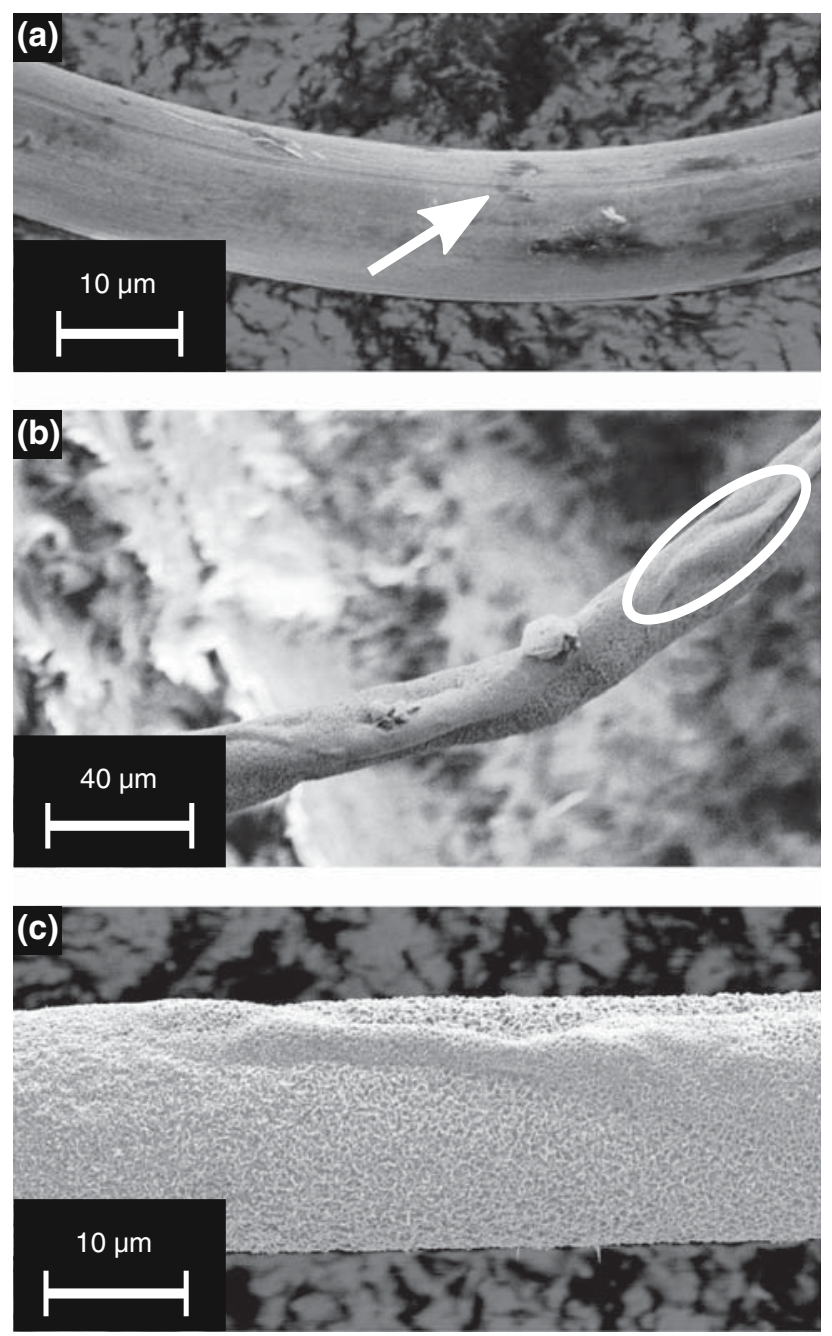

Fig. 4 Scanning electron microscope images of different freestanding $\mathrm{Cu}$ wires. a Pristine wire; $\mathbf{b}, \mathbf{c} \mathrm{Cu}$ wires after EM. See text for details altered as observed in Fig. $4 \mathrm{~b}$, c. The most prominent change is the occurrence of blood-vessel-like structures along the wire, as shown in Fig. $4 \mathrm{~b}$ where one of these structures is marked by an ellipse. Besides this, we often see a characteristic bending of the wire in the middle of its free-standing part where in some cases a constriction appears in the center. We assume that the final electrical cutoff has occurred in the region of the constriction. Moreover, the surface has become rough as can be seen in the enlarged SEM picture in Fig. 4c. The change of the surface roughness is tentatively attributed to the formation of copper oxide at the surface due to local heating, whereas the blood-vessel-like structure and the bending could be caused by thermal expansion arising from the EM process.

To gain further insight into the inner structure of the wire, we took cross-sectional SEM images of a pristine wire (Fig. 5a) and a wire after EM (Fig. 5b). The pristine wire shows a featureless cross-sectional area where only a few scratches from the polishing process are visible. On the

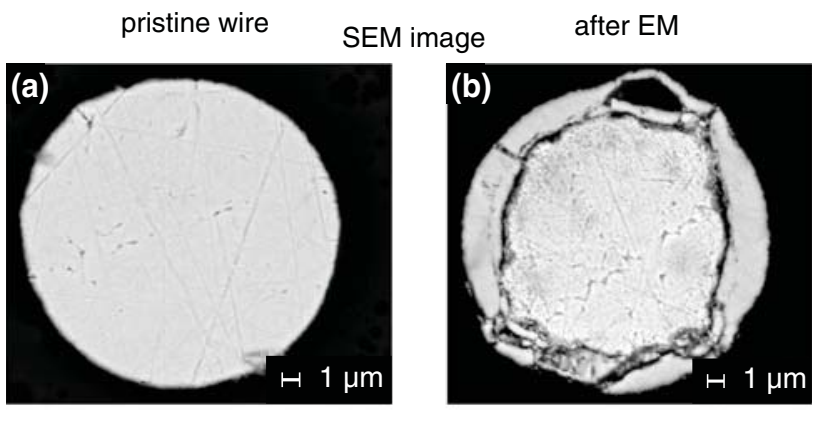

Copper content
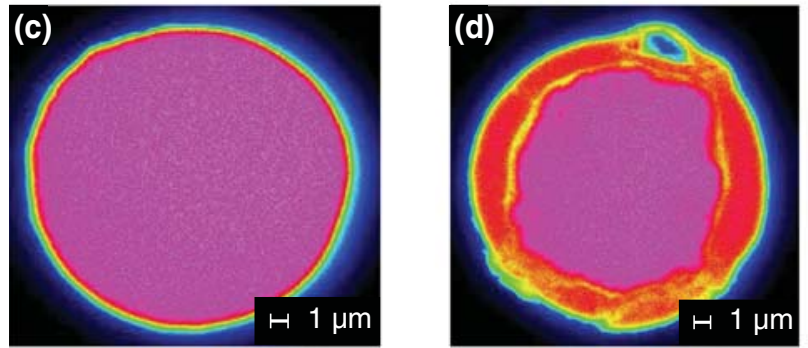

Oxygen content
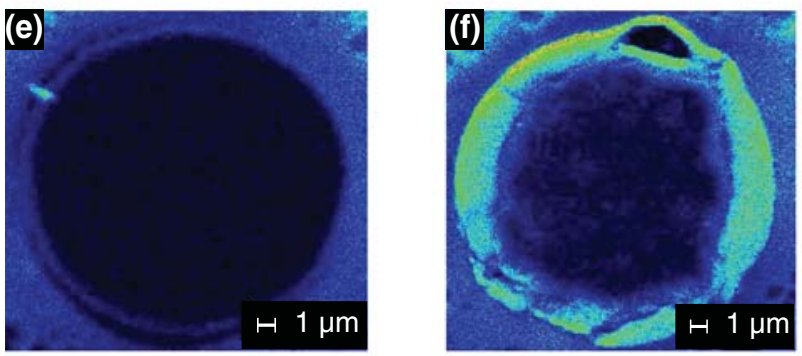

100 at. $\%$

Fig. 5 Cross-sectional analyses of 15- $\mu \mathrm{m}$-diameter copper wires pristine (left panels) and after EM (right panels). a, b SEM images. cf Microprobe images of the wires. Details are given in the text 
contrary, the wire subjected to EM has substantially altered, being divided into two concentric parts. The outer ring appears broken at several positions and displays a large void near the top. Possibly, this void is the cross section of one of the blood-vessel-like structures we observed along the wire, compare Fig. 4b-d. Besides the topographic images, we determined the copper (Fig. 5c, d) and oxygen (Fig. 5e, f) content of the wires by electron microprobe analysis. The untreated wire shows a homogeneous distribution of copper over the full cross section, see Fig. 5c, where the measured percentage of copper is 98.8 at.\%. The oxygen content shown in Fig. $5 \mathrm{c}$ is at the level of only 1.2 at.\%. The two concentric parts of the wire subjected to EM vary in their composition. The element distribution in the inner part is similar to that obtained for the pristine wire, namely 96.6 at.\% of copper and 3.4 at.\% oxygen. In the outer ring-like part, however, a strongly enhanced oxygen content is observed. Here, the composition is 64.5 at.\% of copper and 35.5 at.\% of oxygen. Considering these concentrations and the temperature during the EM (discussed below), we conclude that $\mathrm{Cu}_{2} \mathrm{O}$ was formed. The area displayed in dark on the outer ring of the wire in Fig. 5a, c contains neither copper nor oxygen and, hence, is identified as a void. The separation of the inner and outer ring could be caused by the different thermal expansion coefficients of the core and the shell that formed during EM heating. Indeed, assuming the outer ring to be composed of $\mathrm{Cu}_{2} \mathrm{O}$ the linear thermal expansion coefficients differ by two orders of magnitude between both parts $\left(\alpha_{\mathrm{Cu}}=16.6 \times 10^{-6} \mathrm{~K}^{-1}\right.$ and $\alpha_{\mathrm{Cu}_{2} \mathrm{O}}=$ $23 \times 10^{-8} \mathrm{~K}^{-1}$ at room temperature [20, 21]), leading to huge thermal strains that possibly give rise to delamination upon cooling. The effect of various coatings on the diffusion and the mechanical strain in EM has been addressed in earlier studies for $\mathrm{Cu}$ interconnects [2].

In order to check the decisive role of oxidation on the controlled EM process, we performed similar experiments under UHV. Here no controlled irreversible increase of the resistance upon ramping the voltage was observed until sudden melting. In line with this finding, controlled EM could not be performed on free-standing Au wires, neither under ambient atmosphere nor under UHV. This is in contrast to previous reports where EM on microstructured $\mathrm{Au}$ contacts in contact with a substrate has been successfully performed with various methods [4, 22-24]. In the present case of free-standing wires, where the generated heat cannot be carried away by a substrate, both mechanisms, EM and oxidation, seem to play a role for the resistance increase observed during EM cycles. While EM is at work in atmosphere as well as in vacuum, stabilization by formation of an oxide layer only appears under atmosphere. Systematic EM studies at various $\mathrm{O}_{2}$ pressures while monitoring the temperature are desirable to obtain full optimization and understanding of the EM process for thin free-standing $\mathrm{Cu}$ wires in atmosphere.

\subsection{Heat transport}

Apart from the partial oxidation of the $\mathrm{Cu}$ wires electromigrated in air demonstrated above, the convective heat transport in air might play a role when explaining the striking difference between the result of EM of free-standing $\mathrm{Cu}$ wires in air and under UHV. The importance of the heat transfer via thermal conductance was also demonstrated for thin-film samples deposited on $\mathrm{Si}$ with varying oxide barriers [25]. For an estimate of the temperature distribution along a free-standing $\mathrm{Cu}$ wire subject to Joule heating, we have performed a numerical calculation by the finite element method (FEM) [26]. The steadystate heat conduction equation was numerically solved by taking into account Joule heating, heat conduction along the wire, and convective and radiative losses. Standard material parameters for $\mathrm{Cu}$ were used, in particular a linear temperature dependence of the resistivity at room temperature and above, $\rho(T)=\rho_{0}\left(1+\beta\left(T-T_{0}\right)\right)$ with $\beta=$ $3.9 \times 10^{-3} \mathrm{~K}^{-1}$ [27] and $\rho_{0}=1.7 \mu \Omega \mathrm{cm}$. The temperature at both ends of the 7-mm-long free-standing $\mathrm{Cu}$ wire was held constant at $T=292 \mathrm{~K}$. For a given current density, we obtained the temperature distribution $T(x)$ along the wire axis, the maximum temperature $T_{\max }$ in the center of the free-standing wire at $x=3.5 \mathrm{~mm}$, and the voltage drop between both ends of the wire.

Figure 6 shows the results of the FEM simulation for $\mathrm{EM}$ of a free-standing $\mathrm{Cu}$ wire in air and in vacuum under steady-state conditions. The current-voltage characteristics

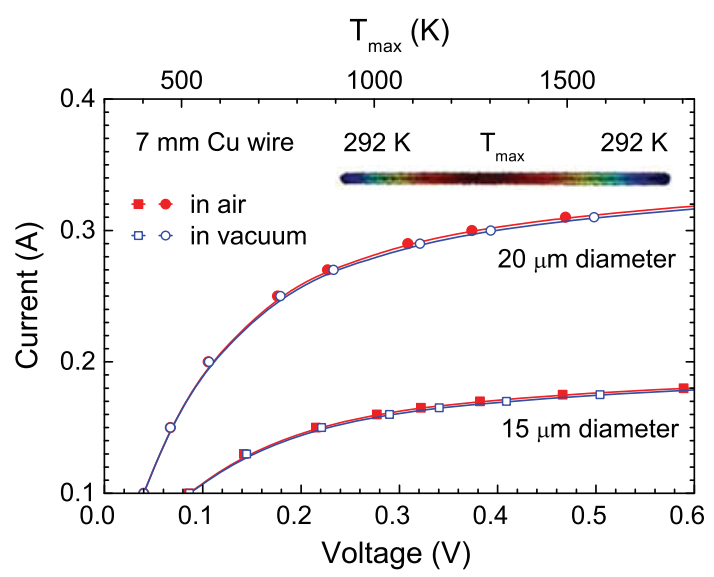

Fig. 6 Current-voltage characteristics of 7-mm-long free-standing $\mathrm{Cu}$ wires of different diameters obtained from FEM simulations in the steady state. $T_{\max }$ is the maximum temperature in the center of the wire. The temperature at both ends of the wire in contact with the sample holder was kept constant at $292 \mathrm{~K}$ 
of two wires of 15 and $20 \mu \mathrm{m}$ thickness show a behavior which is very similar to the experimental case before EM (Fig. 1, region $\mathrm{A}$ of reversible conductance changes) without any free parameter. The simulation, of course, does not deal with the irreversible increase of the resistance due to the onset of EM, i.e., thinning and melting of the wire. Therefore, the simulation continues to temperatures above the melting point of copper $T_{\mathrm{m}} \approx 1360 \mathrm{~K}$ [20]. Nonetheless, the simulation clearly shows that even at temperatures close to the melting point, convective heat transfer and radiation losses do not considerably affect the currentvoltage characteristics. In the standard protocol of controlled EM ramping the current, the temperature increase might be slightly smaller than calculated above for steadystate conditions. However, this does not affect the above conclusion. Regardless of whether EM is performed in air or in vacuum, heat is carried away predominantly through thermal conduction along the wire. Thus, the major effect enabling controlled EM of free-standing $\mathrm{Cu}$ wires seems to be the stabilization of the wire by the copper oxide layer formed in air.

\section{Conclusion}

In conclusion, controlled EM on macroscopic free-standing $\mathrm{Cu}$ wires under ambient conditions results in a gradual thinning of the wires reaching conductances down to a few conductance quanta. SEM and microprobe characterization indicate that the process entails the formation of a copper oxide layer stabilizing the structure mechanically. This conjecture is supported by observing that neither EM control of free-standing $\mathrm{Cu}$ wires under UHV conditions nor EM control of free-standing $\mathrm{Au}$ wires in air and under UHV conditions was possible. The simulation of the current-voltage characteristics obtained by FEM corroborates that the different EM behavior under ambient or UHV conditions is not due to different heat losses by convection and radiation and thus supports the decisive role of copper oxide forming during and stabilizing controlled EM.

Acknowledgements We thank S. Schneider for help with the SEM and C. Pérez León for useful discussions. M. Marz is grateful for support by the Alexander von Humboldt Foundation (Alexander von Humboldt-Stiftung). This work was supported by the ERC Starting Grant NANOCONTACTS (No. 239838) and by the Ministry of Science and Arts (BW), in the framework of its Schlieben-Lange program (Ministerium für Wissenschaft, Forschung und Kunst Baden-Württemberg).

\section{References}

1. J. Black, IEEE Trans. Electron Dev. 16, 338 (1969)

2. C.S. Hau-Riege, Microelectron. Reliab. 44, 195 (2004)

3. D.R. Strachan, D.E. Smith, D.E. Johnston, T.H. Park, M.J. Therien, D.A. Bonnell, A.T. Johnson, Appl. Phys. Lett. 86(4), 043109 (2005)

4. R. Hoffmann, D. Weissenberger, J. Hawecker, D. Stöffler, Appl. Phys. Lett. 93(4), 043118 (2008)

5. H. Park, J. Park, A.K.L. Lim, E.H. Anderson, A.P. Alivisatos, P.L. McEuen, Nature 407(6800), 57 (2000)

6. S. Thiele, F. Balestro, R. Ballou, S. Klyatskaya, M. Ruben, W. Wernsdorfer, Science 344(6188), 1135 (2014)

7. G. Esen, M.S. Fuhrer, Appl. Phys. Lett. 87(26), 263101 (2005)

8. D. Stöffler, S. Fostner, P. Grütter, R. Hoffmann-Vogel, Phys. Rev. B 85(3), 033404 (2012)

9. N. Agrait, A.L. Yeyati, J.M. van Ruitenbeek, Phys. Rep. 377(23), 81 (2003)

10. K. Hansen, E. Laegsgaard, I. Stensgaard, F. Besenbacher, Phys. Rev. B 56, 2208 (1997)

11. D.J. Bakker, Y. Noat, A.I. Yanson, J.M. van Ruitenbeek, Phys. Rev. B 65, 235416 (2002)

12. M. Kiguchi, T. Konishi, S. Miura, K. Murakoshi, Nanotechnology 18, 424011 (2007)

13. I.K. Yanson, O.I. Shklyarevskii, S. Csonka, H. Kempen, S. Speller, A.I. Yanson, J.M. van Ruitenbeek, Phys. Rev. Lett. 95, 256806 (2005)

14. Y. Kurui, Y. Oshima, K. Takayanagi, J. Phys. Soc. Jpn. 76, 123601 (2007)

15. R. de Orio, H. Ceric, S. Selberherr, Microelectron. Reliab. 50(6), 775 (2010)

16. R. Kirchheim, Acta Metall. Mater. 40, 309 (1992)

17. M.A. Korhonen, P. Borgesen, K.N. Tu, C.Y. Li, J. Appl. Phys. 73, 3790 (1993)

18. C.K. Hu, L. Gignac, E. Liniger, B. Herbst, D.L. Rath, S.T. Chen, S. Kaldor, A. Simon, W.T. Tseng, Appl. Phys. Lett. 83, 869 (2003)

19. Z.S. Choi, R. Mönig, C.V. Thompson, J. Appl. Phys. 102, 083509 (2007)

20. R.C. Weast, M.J. Astle, W.H. Beyer, CRC Handbook of Chemistry and Physics (CRC Press, Boca Raton, 2005)

21. O. Madelung, U. Rössler, M. Schulz, SpringerMaterials; sm_lbs_978-3-540-31360-1_68 (Springer, Heidelberg, 1998)

22. Z.M. Wu, M. Steinacher, R. Huber, M. Calame, S.J. van der Molen, C. Schönenberger, Appl. Phys. Lett. 91(5), 053118 (2007)

23. J.M. Campbell, R.G. Knobel, Appl. Phys. Lett. 102(2), 023105 (2013)

24. Y. Kanamaru, M. Ando, J. Shirakashi, J. Vac. Sci. Technol. B 33(2), 02B106 (2015)

25. B. Kießig, R. Schäfer, H.v. Löhneysen, New J. Phys. 16(1), 013017 (2014)

26. Comsol Inc., COMSOL Multiphysics 4.3a. https://www.comsol. com

27. D.C. Giancoli, Physics: Principles with Applications, 3rd edn. (Prentice-Hall International, London, 1991) 